VOL. $66(2002) \quad[259-265]$

\title{
THE WEIGHTED TURÁN TYPE INEQUALITY FOR GENERALISED JACOBI WEIGHTS
}

\author{
J.L. WANG AND S.P. ZHOU
}

We study the weighted Turán type inequality for generalised Jacobi weights, and give a complete positive answer to Zhou's conjecture.

Let $H_{n}$ be the class of real algebraic polynomials of degree $n$, whose zeros all lie in the interval $[-1,1]$. Define

$$
\begin{aligned}
\|f\| & =\max _{-1 \leqslant x \leqslant 1}|f(x)|, \\
\|f\|_{L^{p}} & =\left(\int_{-1}^{1}|f(x)|^{p} d x\right)^{1 / p}, \quad 0<p<\infty .
\end{aligned}
$$

In 1939, Turán [5] established an inequality which was later referred as Turán's inequality. Precisely, Turán proved that for $f \in H_{n},\left\|f^{\prime}\right\| \geqslant C \sqrt{n}\|f\|$.

This inequality was studied quite extensively (interested readers could find useful information in a survey paper [4]). It was generalised to $L^{p}$ spaces, $0<p<\infty$ (see $[6,7,8,10,11,12])$, and its optimal constants were estimated (see $[1,2,3,6,7,8])$.

Note the following fact: If $\Phi_{n}$ is an orthogonal polynomial system on $[-1,1]$ with respect to a weight function $W(x)$, then all zeros of any function in $\Phi_{n}$ lie in the interval $(-1,1)$. To consider the potential application of Turán type inequality to orthogonal polynomial systems, we first should generalise it to the weighted case. For this reason, Zhou in [9] raises the following conjecture.

Conjecture. Let $f \in H_{n}$, then for $0<p<\infty$ and some important weight functions $W(x)$ the inequality

$$
\left\|f^{\prime} W\right\|_{L^{p}} \geqslant C_{W} \sqrt{n}\|f W\|_{L^{p}}
$$

holds for sufficiently large $n$, where the constant $C_{W}>0$ depends upon $W(x)$ and $p$ (in case $p \rightarrow 0$ ) only.

Xiao and Zhou [9] considered some general weight functions and the uniform norm to established the following

Received 21st February, 2002

The author was supported in part by National and Zhejiang Provincial Natural Science Foundations of China and also by Ningbo Key Doctoral Funds.

Copyright Clearance Centre, Inc. Serial-fee code: 0004-9727/02 \$A2.00+0.00. 
THEOREM 1. Let $W(x)$ be a nonnegative continuous piecewise monotone function on the interval $[-1,1]$. If $f \in H_{n}$, then there exists a positive constant $C_{W}$ only depending upon $W(x)$ such that

$$
\left\|f^{\prime} W\right\| \geqslant C_{W} \sqrt{n}\|f W\|
$$

holds for sufficiently large $n$.

This paper will study the weighted Turán type inequality in general $L^{p}$ spaces for $0<p<\infty$. With a quite delicate approach, we shall prove the conjecture for a large group of important weights including Jacobi weights.

In the sequel, we always assume that a weight function $W(x) \geq 0$ satisfies $\int_{-1}^{1} W(x) d x<\infty$ and $W\left(x_{1}\right) \approx W\left(x_{2}\right)$ for any $-1<x_{1}<x_{2} \leq 0$ and $\left|x_{2}-x_{1}\right|<1+x_{1}$ or for any $0<x_{2}<x_{1} \leq 1$ and $\left|x_{2}-x_{1}\right|<1-x_{1}$. Such a function is called a Generalised Jacobi Weight. ( $W\left(x_{1}\right) \approx W\left(x_{2}\right)$ means that there is a constant $M \geq 1$ ( $M$ depends on $W(x))$ such that $M^{-1} W\left(x_{1}\right) \leq W\left(x_{2}\right) \leq M W\left(x_{1}\right)$.)

We see that if $W(x)$ is a Generalised Jacobi Weight, then $W(x)$ may only have a zero or infinity point at the endpoints \pm 1 . It is easy to check that all Jacobi weights $W(x)=(1+x)^{\alpha}(1-x)^{\beta}, \alpha, \beta>-1$, are Generalised Jacobi Weights.

Now we give our main theorem.

Theorem 2. Let $W(x)$ be a Generalised Jacobi Weight, and let $0<p<\infty$. If $f \in H_{n}$, then there exists a positive constant $C_{W, p}$ only depending upon $W(x)$ and $p$ (in case $p \rightarrow 0$ ) such that, for $n$ sufficiently large

$$
\left(\int_{-1}^{1}\left|f^{\prime}(x)\right|^{p} W(x) d x\right)^{1 / p} \geqslant C_{W, p} \sqrt{n}\left(\int_{-1}^{1}|f(x)|^{p} W(x) d x\right)^{1 / p} .
$$

Denote by $-1 \leqslant x_{1}<x_{2}<\cdots<x_{k} \leqslant 1$ all the distinct zeros of $f \in H_{n}$ and by $l_{i}$ the multiplicity of $x_{i}, 1 \leqslant i \leqslant k$. Let $\alpha_{j}$ be the maximum point of $|f(x)|$ between $\left(x_{j}, x_{j+1}\right), 1 \leqslant j<k$. Obviously, for $x \in\left[x_{j}, \alpha_{j}\right]$ (or $x \in\left[\alpha_{j}, x_{j+1}\right]$ ) $f(x)$ is increasing (or decreasing). Set

$$
\begin{aligned}
m(x) & =\frac{f^{\prime}(x)}{f(x)}=\sum_{i=1}^{k} \frac{l_{i}}{x-x_{i}} \\
d_{j} & =\left|m^{\prime}\left(\alpha_{j}\right)\right|^{-1}
\end{aligned}
$$

For $x \in[-1,1]$, it is easy to show that

$$
\begin{aligned}
& \left|m^{\prime}(x)\right|=\sum_{i=1}^{k} \frac{l_{i}}{\left(x-x_{i}\right)^{2}} \geqslant \frac{n}{4}, \\
& \left|m^{\prime}(x)\right| \geqslant\left(x-x_{i}\right)^{-2}, \quad 1 \leqslant i \leqslant k,
\end{aligned}
$$


so that

$$
\sqrt{d_{j}} \leqslant \min _{1 \leqslant i \leqslant k}\left\{\left|\alpha_{j}-x_{i}\right|, 2 n^{-1 / 2}\right\}
$$

We estimate $m(x)$. In the sequel, assume all the inequalities hold for sufficiently large $n$ if not specified.

LEMMA 1 . If $x \in\left(x_{j}, \alpha_{j}-\left(\sqrt{d_{j}} / 8\right)\right] \cup\left[\alpha_{j}+\left(\sqrt{d_{j}} / 8\right), x_{j+1}\right)$, then

$$
|m(x)| \geqslant \frac{2}{25} \frac{1}{\sqrt{d_{j}}}
$$

and if $x \in\left[\alpha_{j}-\left(\sqrt{d_{j}} / 4\right), \alpha_{j}+\left(\sqrt{d_{j}} / 4\right)\right]$, then

$$
|m(x)| \leqslant \frac{4}{9} \frac{1}{\sqrt{d_{j}}}
$$

Proof: For $x \in\left[\alpha_{j}-\left(\sqrt{d_{j}} / 4\right), \alpha_{j}+\left(\sqrt{d_{j}} / 4\right)\right], i=1,2, \cdots, k$, from (3) we have

$$
\frac{3}{4}\left|x_{i}-\alpha_{j}\right| \leqslant\left|x_{i}-\alpha_{j}\right|-\left(\sqrt{d_{j}} / 4\right) \leqslant\left|x_{i}-x\right| \leqslant\left|x_{i}-\alpha_{j}\right|+\frac{\sqrt{d_{j}}}{4} \leqslant \frac{5}{4}\left|x_{i}-\alpha_{j}\right|,
$$

thus by summing up all the terms we get

$$
\frac{16}{25}\left|m^{\prime}\left(\alpha_{j}\right)\right| \leqslant\left|m^{\prime}(x)\right| \leqslant \frac{16}{9}\left|m^{\prime}\left(\alpha_{j}\right)\right| .
$$

Because $m^{\prime}(x)<0, m\left(\alpha_{j}\right)=0$, we have

$$
\begin{gathered}
\left|m\left(\alpha_{j} \pm \frac{\sqrt{d_{j}}}{4}\right)\right|=\left|\int_{\alpha_{j} \pm\left(\sqrt{d_{j}} / 4\right)}^{\alpha_{j}} m^{\prime}(x) d x\right| \leqslant \frac{16}{9}\left|m^{\prime}\left(\alpha_{j}\right)\right| \frac{\sqrt{d_{j}}}{4}=\frac{4}{9} d_{j}^{-1 / 2}, \\
\left|m\left(\alpha_{j} \pm\left(\sqrt{d_{j}} / 8\right)\right)\right|=\left|\int_{\alpha_{j} \pm\left(\sqrt{d_{j}} / 8\right)}^{\alpha_{j}} m^{\prime}(x) d x\right| \geqslant \frac{16}{25}\left|m^{\prime}\left(\alpha_{j}\right)\right| \frac{\sqrt{d_{j}}}{8}=\frac{2}{25} d_{j}^{-1 / 2} .
\end{gathered}
$$

Noting that for $x \in\left(x_{j}, x_{j+1}\right), m(x)$ is decreasing and $m\left(\alpha_{j}\right)=0$, from the above inequalities we obtain Lemma 1 .

We divide the proof of Theorem 2 into the following three lemmas.

Lemma 2. For $j=1,2, \cdots, k-1$, we have

$$
\int_{x_{j}}^{\alpha_{j}}\left|f^{\prime}(x)\right|^{p} W(x) d x \geqslant \frac{1}{2 M^{2}}\left(\frac{1}{50}\right)^{p} n^{p / 2} \int_{x_{j}}^{\alpha_{j}}|f(x)|^{p} W(x) d x
$$


where $M \geq 1$ is the constant appearing in the definition of Generalised Jacobi Weights.

Proof: From (4) we have

$$
\begin{aligned}
\int_{x_{j}}^{\alpha_{j}-\left(\sqrt{d_{j}} / 4\right)}\left|f^{\prime}(x)\right|^{p} W(x) d x & =\int_{x_{j}}^{\alpha_{j}-\left(\sqrt{d_{j}} / 4\right)}|f(x)|^{p}|m(x)|^{p} W(x) d x \\
& \geqslant\left(\frac{2}{25}{\sqrt{d_{j}}}^{-1}\right)^{p} \int_{x_{j}}^{\alpha_{j}-\left(\sqrt{d_{j}} / 4\right)}|f(x)|^{p} W(x) d x \\
& \geqslant\left(\frac{1}{25}\right)^{p} n^{p / 2} \int_{x_{j}}^{\alpha_{j}-\left(\sqrt{d_{j}} / 4\right)}|f(x)|^{p} W(x) d x \text {. (by (2)) }
\end{aligned}
$$

(6)

For the interval $\left[\alpha_{j}-\left(\sqrt{d_{j}} / 4\right), \alpha_{j}\right]$ we consider three cases.

CASE 1. $\alpha_{j} \leqslant 0$.

In this case, for $x \in\left[\alpha_{j}-\left(\sqrt{d_{j}} / 4\right), \alpha_{j}\right] \subset\left[x_{j}, 0\right]$, we see that $0 \leqslant \alpha_{j}-x$ $\leqslant\left(\sqrt{d_{j}} / 4\right) \leqslant \alpha_{j}-\left(\sqrt{d_{j}} / 4\right)-x_{j} \leq x+1$ (by $\left.(3)\right)$, thus have $W(x) \approx W\left(\alpha_{j}\right)$.

SuBCASE 1.1. $\left|f\left(\alpha_{j}-\left(\sqrt{d_{j}} / 4\right)\right)\right| \geqslant\left|f\left(\alpha_{j}\right)\right| / 2$.

Then, for $x \in\left[\alpha_{j}-\left(\sqrt{d_{j}} / 4\right), \alpha_{j}\right],|f(x)| \geqslant\left|f\left(\alpha_{j}\right)\right| / 2$, applying (4), in a similar way to (6) we have

$$
\begin{aligned}
\int_{\alpha_{j}-\left(\sqrt{d_{j}} / 4\right)}^{\alpha_{j}-\left(\sqrt{d_{j}} / 8\right)}\left|f^{\prime}(x)\right|^{p} W(x) d x & \geq \int_{\alpha_{j}-\left(\sqrt{d_{j}} / 4\right)}^{\alpha_{j}-\left(\sqrt{d_{j}} / 8\right)}|f(x)|^{p}|m(x)|^{p} W(x) d x \\
& \geq \frac{1}{M}\left(\frac{1}{50}\right)^{p} n^{p / 2}\left|f\left(\alpha_{j}\right)\right|^{p} W\left(\alpha_{j}\right) \frac{\sqrt{d_{j}}}{8} \\
& \geq \frac{1}{2 M^{2}}\left(\frac{1}{50}\right)^{p} n^{p / 2} \int_{\alpha_{j}-\left(\sqrt{d_{j}} / 4\right)}^{\alpha_{j}}|f(x)|^{p} W(x) d x .
\end{aligned}
$$

SubCASE 1.2. $\left|f\left(\alpha_{j}-\left(\sqrt{d_{j}} / 4\right)\right)\right|<\left|f\left(\alpha_{j}\right)\right| / 2$.

We first assume $0<p<1$. Now that $p-1<0$, from (5) we have

$$
\begin{aligned}
\int_{\alpha_{j}-}^{\alpha_{j}} & =f_{\left.d_{j} / 4\right)}\left|f^{\prime}(x)\right|^{p} W(x) d x \\
& =\int_{\alpha_{j}-\left(\sqrt{d_{j}} / 4\right)}^{\alpha_{j}}|f(x)|^{p-1}|m(x)|^{p-1}\left|f^{\prime}(x)\right| W(x) d x \\
& \geqslant\left(\frac{4}{9}{\sqrt{d_{j}}}^{-1}\right)^{p-1} \frac{W\left(\alpha_{j}\right)}{M}\left|f\left(\alpha_{j}\right)\right|^{p-1} \int_{\alpha_{j}-\left(\sqrt{d_{j}} / 4\right)}^{\alpha_{j}}\left|f^{\prime}(x)\right| d x
\end{aligned}
$$




$$
\begin{aligned}
& \geqslant\left(\frac{4}{9}{\sqrt{d_{j}}}^{-1}\right)^{p-1} \frac{W\left(\alpha_{j}\right)}{M}\left|f\left(\alpha_{j}\right)\right|^{p-1}\left|f\left(\alpha_{j}\right)-f\left(\alpha_{j}-\frac{\sqrt{d_{j}}}{4}\right)\right| \\
& \geq \frac{9}{2}\left(\frac{4}{9}{\sqrt{d_{j}}}^{-1}\right)^{p} M^{-1}\left|f\left(\alpha_{j}\right)\right|^{p} W\left(\alpha_{j}\right) \frac{\sqrt{d_{j}}}{4} \\
& \geqslant M^{-2}\left(\frac{2}{9}\right)^{p} n^{p / 2} \int_{\alpha_{j}-\left(\sqrt{d_{j}} / 4\right)}^{\alpha_{j}}|f(x)|^{p} W(x) d x .
\end{aligned}
$$

In case $1 \leq p<\infty$, by Hölder's inequality we have

$$
\begin{aligned}
\int_{\alpha_{j}-\left(\sqrt{d_{j}} / 4\right)}^{\alpha_{j}}\left|f^{\prime}(x)\right|^{p} W(x) d x & \geqslant \frac{1}{M} W\left(\alpha_{j}\right) \int_{\alpha_{j}-\left(\sqrt{d_{j}} / 4\right)}^{\alpha_{j}}\left|f^{\prime}(x)\right|^{p} d x \\
& \geqslant \frac{1}{M} W\left(\alpha_{j}\right)\left(\frac{\sqrt{d_{j}}}{4}\right)^{-p+1}\left(\int_{\alpha_{j}-\left(\sqrt{d_{j}} / 4\right)}^{\alpha_{j}}\left|f^{\prime}(x)\right| d x\right)^{p} \\
& \geqslant \frac{1}{M}\left(\frac{\sqrt{d_{j}}}{4}\right)^{-p+1}\left(\frac{\left|f\left(\alpha_{j}\right)\right|}{2}\right)^{p} W\left(\alpha_{j}\right) \\
& \geqslant \frac{1}{M} n^{p / 2}\left|f\left(\alpha_{j}\right)\right|^{p} W\left(\alpha_{j}\right) \frac{\sqrt{d_{j}}}{4} \\
& \geqslant \frac{1}{M} n^{p / 2} \int_{\alpha_{j}-\left(\sqrt{d_{j}} / 4\right)}^{\alpha_{j}}|f(x)|^{p} W(x) d x
\end{aligned}
$$

From $(7),(8)$ and $\left(8^{\prime}\right)$, we obtain that

(9) $\quad \int_{\alpha_{j}-\left(\sqrt{d_{j}} / 4\right)}^{\alpha_{j}}\left|f^{\prime}(x)\right|^{p} W(x) d x \geqslant \frac{1}{2 M^{2}}\left(\frac{1}{50}\right)^{p} n^{p / 2} \int_{\alpha_{j}-\left(\sqrt{d_{j}} / 4\right)}^{\alpha_{j}}|f(x)|^{p} W(x) d x$.

CASE 2. $x_{j} \geqslant 0$.

In this case, $x \in\left[\alpha_{j}-\left(\sqrt{d_{j}} / 4\right), \alpha_{j}\right] \subset\left[x_{j}, \alpha_{j}\right] \subset[0,1]$, thus $\alpha_{j}-x \leqslant\left(\sqrt{d_{j}} / 4\right)$ $\leqslant x_{j+1}-\alpha_{j} \leqslant 1-\alpha_{j}$ (by (3)), so $W(x) \approx W\left(\alpha_{j}\right)$. With a similar way to Case 1 , we reach (9) as well.

CAse 3. $x_{j}<0<\alpha_{j}$.

When $\alpha_{j} \leqslant\left(\sqrt{d_{j}} / 4\right)$, we see that $\left[\alpha_{j}-\left(\sqrt{d_{j}} / 4\right), \alpha_{j}\right]$ $\subset\left[-\left(\sqrt{d_{j}} / 4\right),\left(\sqrt{d_{j}} / 4\right)\right] \subset[-(1 / 2 \sqrt{n}),(1 / 2 \sqrt{n})] \subset[-1 / 2,1 / 2]$. From the definition of Generalised Jacobi Weights, for $x \in\left[\alpha_{j}-\left(\sqrt{d_{j}} / 4\right), \alpha_{j}\right]$, we surely have $W(x) \approx W\left(\alpha_{j}\right)$.

When $\alpha_{j}>\left(\sqrt{d_{j}} / 4\right)$, then $\left[\alpha_{j}-\left(\sqrt{d_{j}} / 4\right), \alpha_{j}\right] \subset\left[0, \alpha_{j}\right] \subset\left[0, x_{j+1}\right] \subset[0,1]$. for $x \in\left[\alpha_{j}-\left(\sqrt{d_{j}} / 4\right), \alpha_{j}\right], \alpha_{j}-x \leqslant\left(\sqrt{d_{j}} / 4\right) \leqslant x_{j+1}-\alpha_{j}<1-\alpha_{j}$ (see (3)), thus we also have the relation $W(x) \approx W\left(\alpha_{j}\right)$. 
A similar argument to Case 1 leads to (9) in case 3.

Combining the conclusions of the above three cases with (6), we have finished the proof of Lemma 2.

By the same technique, we have

LEMMA 3. For $j=1,2, \cdots, k-1$, we have

$$
\int_{\alpha_{j}}^{x_{j+1}}\left|f^{\prime}(x)\right|^{p} W(x) d x \geqslant \frac{1}{2 M^{2}}\left(\frac{1}{50}\right)^{p} n^{p / 2} \int_{\alpha_{j}}^{x_{j+1}}|f(x)|^{p} W(x) d x .
$$

If $-1<x_{1}$ or $x_{k}<1$, for $x \in\left[-1, x_{1}\right)$ or $x \in\left(x_{k}, 1\right]$, it is easy to see

$$
|m(x)|=\left|\sum_{i=1}^{k} \frac{l_{i}}{x-x_{i}}\right| \geqslant n / 2
$$

thus we have the next lemma.

LEMMA 4. If $f(-1) \neq 0$, then

$$
\int_{-1}^{x_{1}}\left|f^{\prime}(x)\right|^{p} W(x) d x \geqslant\left(\frac{n}{2}\right)^{p} \int_{-1}^{x_{1}}|f(x)|^{p} W(x) d x
$$

if $f(1) \neq 0$, then

$$
\int_{x_{k}}^{1}\left|f^{\prime}(x)\right|^{p} W(x) d x \geqslant\left(\frac{n}{2}\right)^{p} \int_{x_{k}}^{1}|f(x)|^{p} W(x) d x
$$

From Lemma 2 to Lemma 4, the proof of the inequality (1) is completed. Therefore we have finished Theorem 2 .

REMARK 1. In fact, the constant $C_{W, p}$ in Theorem 2 can be taken as $\left.\left(\left(2^{-1} M^{-2}\right)\right)^{1 / p}\right) / 50$ for $0<p<1$, and $M^{-2} / 100$ for $1 \leq p<\infty$.

REMARK 2. With $f(x)=\left(1-x^{2}\right)^{[n / 2]}$, we see that the order $n^{1 / 2}$ in Theorem 2 can not be improved for Jacobi weights $(1-x)^{\alpha}(1+x)^{\beta}, \alpha, \beta>-1$.

\section{REFERENCES}

[1] V.F. Bakenko and S.A. Pichugov, 'Accurate inequality for the derivative of trigonometric polynomials which have real zeros', (in Russian), Mat. Zametki 39 (1986), 330-336.

[2] V.F. Bakenko and S.A.Pichugov, 'Inequality for the derivative of polynomials with real zeros', (in Russian), Ukrain. Math. Zh. 38 (1986), 411-416.

[3] J. Eröd, 'Bizonyos polinomok maximumairól', Math. Fiz. Lapok 46 (1939), 58-82. 
[4] G.V. Milovanović and T.M. Rassias, 'New developments on Turán's extremal problems for polynomials', in Approximation Theory: In Memory of A. K. Varma (Marcel Dekker, Inc., New York, 1998), pp. 433-447.

[5] P. Turán, 'Uber dir ableitung von polynomen', Compositio Math. 7 (1939), 89-95.

[6] A.K. Varma, 'An analogue of some inequality of P. Turán concerning algebraic polynomials satisfying certain conditions', Proc. Amer. Math. Soc 55 (1976), 305-309.

[7] A.K. Varma, 'An analogue of some inequality of P. Turán concerning algebraic polynomials having all zeros inside $[-1,1]$ ', Proc. Amer. Math. Soc 69 (1978), 25-33.

[8] A.K. Varma, 'Some inequalities of algebraic polynomials having all zeros inside $[-1,1]$ ', Proc. Amer. Math. Soc. 88 (1983), 227-233.

[9] W. Xiao and S.P. Zhou, 'On weighted Turán type inequality', Glas. Mat. Ser. III 34(54) (1999), 197-202.

[10] S.P. Zhou, 'Some remark on Turán type inequality', J. Approx. Theory 68 (1992), 45-48.

[11] S.P. Zhou, 'Some remark on Turán type inequality. II', J. Math. Anal. Appl. 180 (1993), 138-143.

[12] S.P. Zhou, 'Some remark on Turán type inequality III: the completion', Anal. Math. 21 (1995), 313-318.

The Mathematical Institute

Ningbo University

Ningbo

Zhejiang 315211

China

and

Department of Mathematics

Shaoxing Arts and Science College

Shaoxing

Zhejiang 312000

China

e-mail: jiaswu@zscas.edu.ch
The Mathematical Institute

Ningbo University

Ningbo

Zhejiang 315211

China. 